\section{Sobreposição de casos novos de hanseníase em redes de convívio domiciliar em dois municípios do Norte e Nordeste do Brasil, 2001-2014}

\section{Overlapping of new leprosy cases in household contact networks in two municipalities in North and Northeast Brazil, 2001-2014}

\author{
Superposición de casos nuevos de lepra en redes \\ de convivencia domiciliaria en dos municipios \\ del norte y nordeste de Brasil, 2001-2014
}

\author{
Adriana da Silva dos Reis 1,2 \\ Eliana Amorim de Souza 3 \\ Anderson Fuentes Ferreira 1 \\ Gilberto Valentim da Silva 4 \\ Suyanne Freire de Macedo 5,6 \\ Olivia Dias de Araújo 7 \\ Jessíca Reco Cruz 8,9 \\ Gabriela Soledad Márdero García 1 \\ Maria Angélica Gomes Carneiro 1 \\ Jaqueline Caracas Barbosa 1,2 \\ Alberto Novaes Ramos Jr. 2,10
}

doi: 10.1590/0102-311X00014419

\author{
Correspondência \\ A. S. Reis \\ Netherlands Hanseniasis Relief do Brasil. \\ Av. Humberto Monte 2929, sala 1109, Torre Norte, Fortaleza, \\ CE 60440-593, Brasil. \\ drikluk@gmail.com

\footnotetext{
1 Programa de Pós-graduação em Saúde Pública, Universidade Federal do Ceará, Fortaleza, Brasil.

2 Netherlands Hanseniasis Relief do Brasil, Fortaleza, Brasil. 3 Instituto Multidisciplinar em Saúde, Universidade Federal da Bahia, Vitória da Conquista, Brasil.

4 Coordenação do Programa de Controle da Hanseníase, Secretaria Municipal de Saúde, Picos, Brasil.

5 Programa de Pós-graduação em Saúde Coletiva, Universidade Estadual do Ceará, Fortaleza, Brasil.

6 Departamento de Enfermagem, Universidade Federal do Piauí, Picos, Brasil.

7 Departamento de Enfermagem, Universidade Federal do Piauí, Teresina, Brasil.

8 Faculdade de Ciências Biomédicas de Cacoal, Cacoal, Brasil. 9 Secretaria Municipal de Saúde de Cacoal, Cacoal, Brasil. 10 Departamento de Saúde Comunitária, Universidade Federal do Ceará, Fortaleza, Brasil.
}

ção da doença em dois municípios brasileiros, um da Região Norte e outro da Nordeste, de 2001 a 2014. Estudo transversal, descritivo e analitico, com base em dados primários e secundários de casos novos de hanseníase em indivíduos residentes em Picos, no Estado do Piaui, e em Rolim de Moura, no Estado de Rondônia. "Sobreposição" foi definida como casos novos com ocorrência de pelo menos mais um caso de hanseníase em sua RCD. Cada RCD foi composta por caso referência (primeiro caso notificado), casos coprevalentes (contatos que se tornaram casos novos) e contatos domiciliares de caso referência. Um total de 437 casos novos abordados pelos projetos INTEGRAHANS Norte-Nordeste e Piauí foram avaliados, 287 (65,7\%) em Picos e 150 (34,3\%) em Rolim de Moura. Do total de casos avaliados, 129 (44,9\%) em Picos e 98 $(65,3 \%)$ em Rolim de Moura relataram a ocorrência de sobreposição. Verificou-se maior frequência de pessoas do sexo feminino em Rolim de Moura $(n=95,63,3 \%)$ e do masculino em Picos ( $n=147,51,2 \%)$; na faixa etária entre 41-60 anos de idade (Rolim de Moura $n=70,46,7 \%$; Picos $n=115$, 40,1\%); com ensino fundamental (Rolim de Moura $n=80,54 \%$; Picos $n=125$, 44,5\%) e que morava no mesmo domicílio com até 3 pessoas em Rolim de Moura $(n=105,70 \%)$ e com mais de 4 pessoas em Picos $(n=287,100 \%)$. A sobreposição de casos de hanseníase em RCD apresentou magnitude considerável nos contextos analisados, demonstrando aspectos de vulnerabilidade ampliada. Essa perspectiva deve ser considerada e integrada às ações de vigilância e controle da hanseníase.

Hanseníase; Monitoramento Epidemiológico; Transmissão de Doenças Infecciosas 


\section{Introdução}

A hanseníase mantém-se como problema de saúde pública, mesmo tratando-se de uma doença curável e com meios para o diagnóstico precoce e tratamento medicamentoso eficaz e gratuito 1 . O Brasil se enquadra em um grupo denominado "países prioritários globais" para o controle da doença. Composto por 22 países, esse grupo detectou 205.304 casos novos em 2016, correspondendo a 95\% da carga global da doença 2. O fato de tratar-se de uma síndrome clínica dermatoneurológica, com potencial evolução para danos permanentes, reforça a elevada transcendência da hanseníase, inclusive por estar fortemente relacionada ao estigma 3.

Com 25.218 notificações em 2016 e coeficiente de detecção de 12,2 casos/100.000 habitantes, o Brasil ocupou o segundo lugar em número de casos novos notificados no mundo (11,7\% dos casos) 2 . Para o ano de 2017 foi observado um aumento no número de pessoas afetadas pela doença no país, com registro de 26.875 casos novos ${ }^{2}$. Portanto, a persistência da hanseníase no Brasil mantém o desafio para seu controle.

Apresenta distribuição heterogênea e focal nos territórios endêmicos. Estudos com análises apoiadas pelo Ministério da Saúde do Brasil destacaram 10 clusters de maior endemicidade localizados nas regiões Centro-oeste, Nordeste e Norte do país, com alta carga da doença 4 . Em diferentes áreas, ao exemplo, a Região Nordeste, é possível reconhecer uma relação direta entre elevada ocorrência de casos novos e contexto social de pobreza, o que dificulta ainda mais o seu controle e reafirma os processos de determinação social a ela vinculados 5 . Como enfermidade integrante do grupo de doenças negligenciadas, tem sua expressão marcadamente relacionada a populações que experienciam diferentes dimensões de vulnerabilidade, e à perpetuação do ciclo de pobreza, desigualdade e exclusão social em várias gerações de uma mesma família 2,6.

Nesse contexto, falhas operacionais de controle nas redes de atenção à saúde possibilitam a manutenção dos elevados níveis de endemicidade da hanseníase no país 7, como ocorre, por exemplo, nos estados do Piauí (Região Nordeste) e de Rondônia (Região Norte) 8. Os municípios de Picos (Piauí) e Rolim de Moura (Rondônia), territórios do presente estudo, estão situados em contextos estaduais com ocorrência de número expressivo de casos novos entre crianças menores de 15 anos de idade 8 . Esse acontecimento reforça a existência de focos ativos de transmissão de Mycobacterium leprae no território 2,3, com elevada dinâmica de circulação, em particular nos contextos domiciliares 9 .

As ações que favorecem o diagnóstico e o tratamento oportuno são reconhecidas como as principais estratégias para o controle 1,3. A vigilância de contatos deve ser priorizada, atentando para a necessidade de sua realização como atividade rotineira e longitudinal na atenção básica em especial 1 . Em particular, pelo fato de a hanseníase ter um longo período de incubação e um risco acrescido para adoecimento das pessoas que convivem e/ou conviveram com um caso ativo da doença. Esse risco pode ser até 14 vezes maior entre contatos intradomiciliares de casos multibacilares e até 2 vezes maior entre os contatos intradomiciliares de casos paucibacilares $3,9,10$.

$\mathrm{O}$ acesso a diagnóstico e tratamento oportunos reduz o sofrimento da pessoa acometida e de sua Rede de Convívio Domiciliar (RCD), e favorece a interrupção da transmissão, principalmente em áreas de maior endemicidade e vulnerabilidade 11. Dessa forma, a cobertura e a qualidade das ações de vigilância e controle de contatos intra e extradomiciliares (contatos sociais) têm sido estratégias importantes para a redução da carga da doença 12.

O Ministério da Saúde, a partir de 2016, ampliou o conceito de contato, como resultado da necessidade de estender as ações de controle. Introduziu-se a categoria de contatos sociais como qualquer pessoa que conviva ou tenha convivido em relações familiares, ou não, de forma próxima e por um tempo prolongado com um caso de hanseníase sem tratamento 1. Entretanto, as estratégias de operacionalização desse conceito nas redes de atenção do Sistema Único de Saúde (SUS) não foram estabelecidas ainda. Dessa forma, torna-se fundamental pensar em estratégias operacionais inovadoras que permitam a integração desse conceito ampliado de contatos 11,12 . Poucos estudos têm sinalizado caminhos potenciais para o alcance de contextos familiares e sociais, como a proposta de indicador baseado na sobreposição de casos da doença em uma mesma RCD, com o acometimento de diferentes gerações destas famílias 11 .

Diante das lacunas existentes para se reconhecer e compreender os aspectos referentes ao controle da hanseníase em diferentes populações de contatos de caso referência, o estudo de RCD e das 
dimensões de vulnerabilidade para a ocorrência da doença tem se mostrado promissor. Investigar essas redes poderá resultar em evidências de cunho operacional que retratam o cenário da endemia em municípios com diferentes graus de endemicidade. Nessa perspectiva, objetivou-se analisar a magnitude da ocorrência e o perfil sociodemográfico de casos de hanseníase vinculados à RCD com sobreposição da doença nos municípios de Picos e Rolim de Moura, no período de 2001 a 2014.

\section{Métodos}

\section{Local de estudo}

Os munícipios do estudo estão localizados nas regiões Norte e Nordeste do país, as quais compõem importantes clusters da hanseníase no Brasil 4 (Figura 1). O Município de Picos tem densidade demográfica de 137,30 habitantes/km² e população estimada para 2018 de 78.002. Rolim de Moura apresenta densidade de 34,74 habitantes $/ \mathrm{km}^{2}$ e população estimada para 2018 de $57.07413,14$. O percentual de cobertura da Estratégia Saúde da Família (ESF) nesses munícipios, para novembro de 2018, foi de $100 \%$ e 78,58\%, respectivamente, para Picos e Rolim de Moura 15 . O Índice de Desenvolvimento Humano (IDH) pelo Censo Demográfico de 2010 em Picos foi de 0,698 e em Rolim de Moura, de 0,700. O Índice de Gini foi de 0,56 e 0,53, respectivamente 16,17,18.

\section{Desenho do estudo}

Estudo transversal com abordagens descritiva e analítica, desenvolvido com base em dados primários e secundários de casos novos de hanseníase notificados em indivíduos residentes em Picos, e em Rolim de Moura. Esses casos novos de hanseníase foram avaliados durante as pesquisas operacionais INTEGRAHANS Norte-Nordeste, coordenada pela Universidade Federal do Ceará (UFC) e INTEGRAHANS-Piauí, coordenada pela Universidade Federal do Piauí (UFPI), tendo parceria da UFC.

\section{Coleta e análise de dados}

Os casos novos que compuseram a população inicial deste trabalho foram identificados por intermédio do Sistema de Informação de Agravos de Notificação (SINAN) do Ministério da Saúde, cuja base foi obtida junto às secretarias estaduais e municipais dos locais de estudo. Os dados do Município de Picos correspondem aos registros do período de 2001 a 2014, e do Município de Rolim de Moura aos registros de 2001 a 2012, tendo em vista os diferentes momentos de realização dos projetos INTEGRAHANS. Foram excluídos todos os casos que tiveram como critério de saída "erro diagnóstico".

Os dados secundários do SINAN foram complementados gerando um novo banco com base nos dados primários obtidos com a avaliação dos casos novos identificados durante a abordagem domiciliar. A participação de cada pesquisado foi efetivada com a oportuna assinatura do Termo de Consentimento Livre Esclarecido e com a aplicação de um instrumento estruturado com variáveis de diferentes naturezas. As variáveis sociodemográficas e econômicas contidas no instrumento incluíam: sexo (masculino e feminino); raça/cor (branca, parda, negra, amarela, indígena); faixa etária em anos $(<15,15-40,41-60,>60)$; situação de moradia (moradia estável definitiva, moradia estável temporária, assentamento, outra); escolaridade (sem estudo, Ensino Fundamental, Ensino Médio, Ensino Superior); número de moradores no domicílio atualmente (0-3, 4 ou mais); renda total do domicílio em salários mínimos ( $\mathrm{R} \$ 954,00:<1,1-2,>2$ ); mudança de renda após o tratamento de hanseníase (não, sim com redução da renda, sim com aumento da renda); e acesso ao programa Bolsa Família (não e sim). Prevendo a possibilidade de ocorrer viés de memória e/ou desconhecimento sobre o momento do diagnóstico e tratamento da hanseníase, utilizou-se como fonte complementar de dados os prontuários médicos, respeitando-se sempre os aspectos éticos vinculados.

Para este trabalho, definiu-se caso referência como o primeiro caso de hanseníase notificado em uma RCD. A Rede é composta por caso referência, caso coprevalente (contato que se tornou caso) e 


\section{Figura 1}

Localização geográfica dos municípios do estudo nos Estados do Piauí (Picos) e de Rondônia (Rolim de Moura), Brasil.

1a) Rondônia
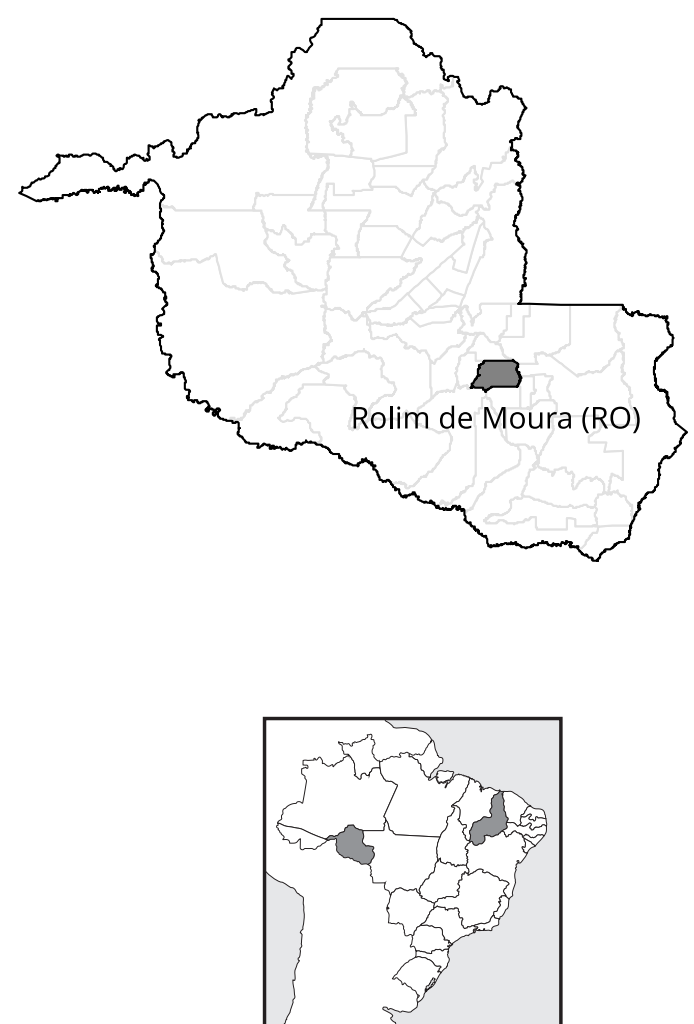

1b) Piauí

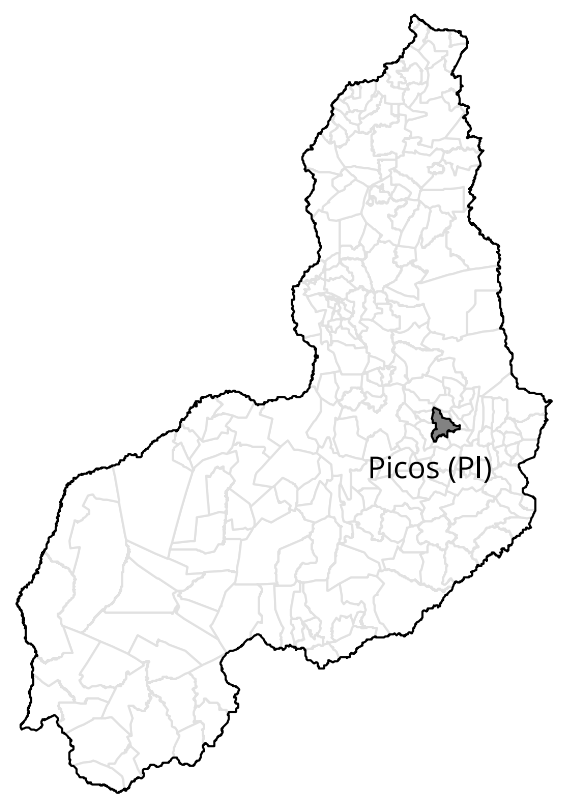

contatos (intradomiciliares e sociais) 11. Foram identificados casos novos que relataram a ocorrência de pelo menos mais um caso de hanseníase em sua RCD, definida neste estudo como "sobreposição".

Para a entrada e análise dos dados foi utilizado o software Epi Info 7.2.1 (https://www.cdc.gov/ epiinfo/index.html) e Stata 11.2 (https://www.stata.com). Os dados descritivos foram apresentados por meio de tabelas com frequências absolutas e relativas. Para a análise bivariada foi considerado como desfecho ter sobreposição de casos em RCD. Os dados das análises bivariadas foram apresentados em tabelas com cálculo das razões de prevalência e seus respectivos intervalos de $95 \%$ de confiança (IC95\%), além do valor de p, considerado significante quando menor que 0,05.

\section{Aspectos éticos}

O projeto foi submetido e aprovado pelo Comitê de Ética em Pesquisa (CEP) da UFC, sob o número 544.962, de 28 de fevereiro de 2014, e da UFPI, sob o número 1.115.818, de 17 de julho de 2015. Respeitou-se a Resolução no 466, de 12 de outubro de 2012, do Conselho Nacional de Saúde. 


\section{Resultados}

Do total de 437 casos novos abordados com base nos projetos INTEGRAHANS Norte-Nordeste e Piauí, e avaliados neste estudo, 51,9\% ( $\mathrm{n}=227)$ referiram ocorrência de sobreposição de casos de hanseníase em sua RCD. O Município de Rolim de Moura apresentou a maior proporção de sobreposição de $\operatorname{casos}(\mathrm{n}=98 ; 65,3 \%)$ em comparação com Picos ( $\mathrm{n}=129 ; 44,9 \%)$ (Figura 2). Verificou-se ainda maior frequência de sobreposição em densidade de pelo menos 1 caso coprevalente tanto em Rolim de Moura ( $n=63,42 \%)$ quanto em Picos $(n=94,32,8 \%)$, em relação aos que negaram a ocorrência deste desfecho.

\section{Figura 2}

População do estudo nos municípios dos Estados do Piauí (Picos) e de Rondônia (Rolim de Moura), Brasil.

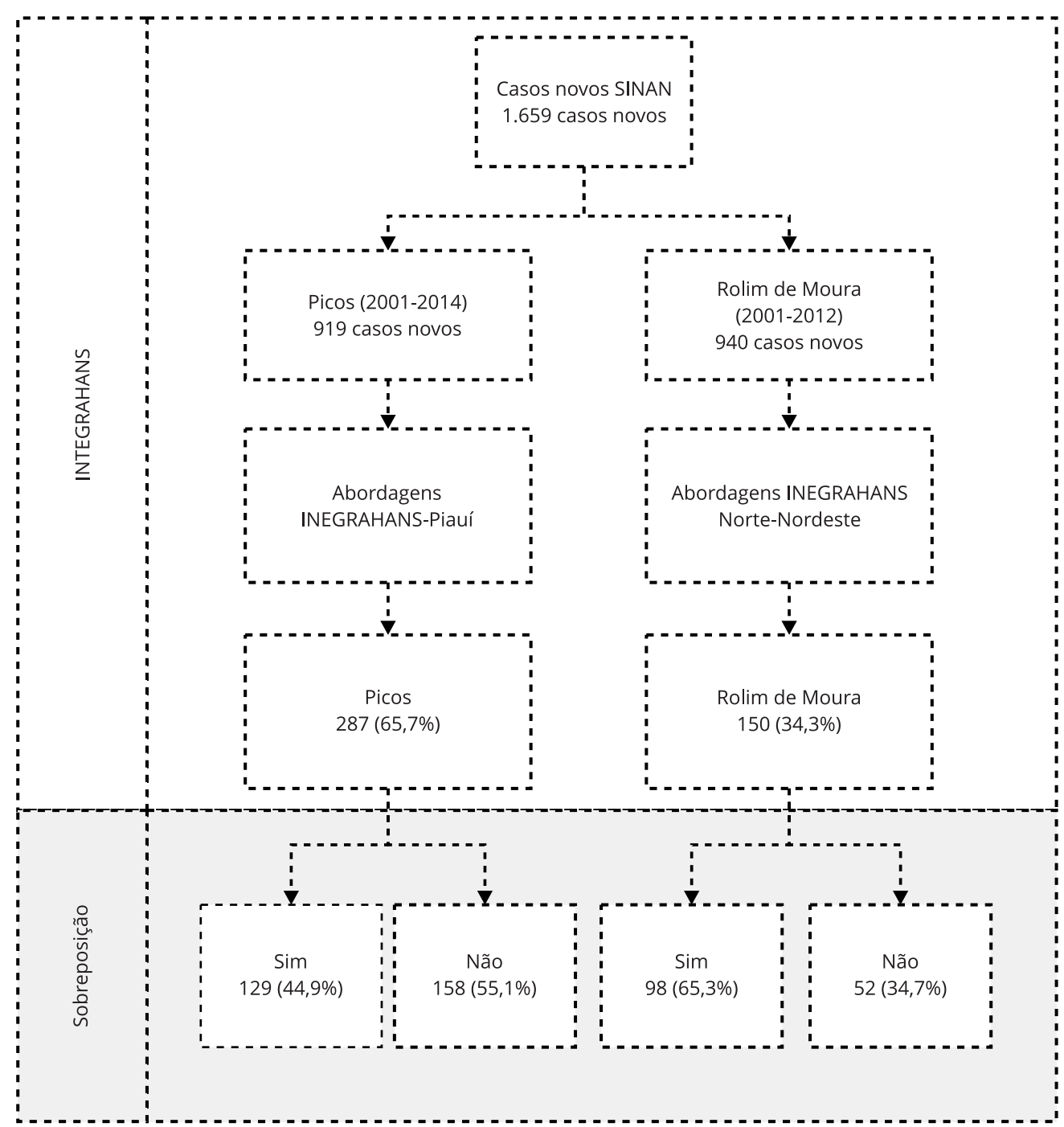

SINAN: Sistema de Informação de Agravos de Notificação. 
Ao caracterizar os casos novos abordados, verificou-se que em Rolim de Moura houve maior frequência de casos do sexo feminino ( $\mathrm{n}=95,63,3 \%$ ) e em Picos, do masculino ( $\mathrm{n}=147,51,2 \%)$. Houve maior ocorrência da raça/cor parda (Rolim de Moura $n=78,52,7 \%$; Picos n =152, 53,3\%); faixa etária 41-60 anos (Rolim de Moura $n=70,46,7 \%$; Picos $n=115,40,1 \%$ ); situação de moradia definitiva (Rolim de Moura n = 136, 91,3\%; Picos n =231, 80,5\%); e escolaridade de Ensino Fundamental (Rolim de Moura $n=80,54 \%$; Picos $n=125,44,5 \%)$. Do ponto de vista social e econômico, foram mais frequentes: número de moradores por domicílios de até 3 pessoas em Rolim de Moura $(\mathrm{n}=105,70 \%)$ e de 4 ou mais pessoas em Picos ( $\mathrm{n}=287,100 \%)$; renda total menor que 1 salário mínimo (Rolim de Moura $\mathrm{n}=68$, 55,7\%; Picos n = 83, 33,9\%); não ter mudança da renda após o diagnóstico da hanseníase (Rolim de Moura n =102, 69,4\%; Picos n =256, 89,2\%); e não ter recebimento do benefício do programa Bolsa Família (Rolim de Moura n =121, 82,3\%; Picos n =188, 65,5\%) (Tabela 1).

A ocorrência de sobreposição esteve associada principalmente à residência em Picos $(\mathrm{n}=129$, 56,8\%), com RP = 1,45 (IC95\%: 1,22-1,73; valor de $\mathrm{p}=0,0001$ ). Ser do sexo feminino foi um fator de proteção ( $R P=$ 0,79; IC95\%: 0,66-0,96; valor de $\mathrm{p}<$ 0,0130) para a ocorrência de sobreposição na RDC. Morar com 4 ou mais pessoas no mesmo domicílio foi significativamente um fator de risco para sobreposição ( $R P=1,27$; IC95\%: 1,05-1,53; valor de $\mathrm{p}<0,0191)$. As demais variáveis analisadas não se mostraram estatisticamente significativas (Tabela 2).

\section{Discussão}

A sobreposição de casos de hanseníase em RCD mostrou-se um evento frequente nos municípios hiperendêmicos avaliados, relacionado principalmente à maior densidade de moradores em um mesmo domicílio. Essas RCD apresentavam vulnerabilidade acrescida diante de um perfil sociodemográfico e econômico desfavorável, amplificando o caráter de doença negligenciada e o impacto da hanseníase em pessoas e suas famílias. A abordagem proposta é inovadora ao investigar a RCD como um evento sentinela em potencial e que pode ser operacionalizada nas ações de vigilância de contatos.

A elevada proporção de casos novos abordados com baixa escolaridade, que dispõem de renda menor que 1 salário mínimo e fazem parte dos critérios estabelecidos para o cadastro no programa Bolsa Família, foi evidenciada neste estudo. O desenvolvimento da pesquisa nas regiões Norte e Nordeste do país, áreas com maior desigualdade social e de grande endemicidade para hanseníase, potencializa a avaliação desta abordagem e pode justificar a elevada ocorrência de sobreposição 11. Para além da vulnerabilidade individual e social, essas áreas podem apresentar condições de maior precariedade da rede de atenção à saúde, com dificuldade de acesso a diagnóstico e tratamento 11, assim como de seguimento e adesão às orientações individuais e coletivas 19 .

Apesar de os municípios apresentarem cobertura satisfatória da ESF, 100\% em Picos e 78,6\% em Rolim de Moura 15, estudos desenvolvidos nestes municípios apontam para a qualidade regular no acompanhamento às pessoas diagnosticadas com hanseníase durante todo tratamento, incluindo o reconhecimento da fragilidade na vigilância de contatos 11,20. Esse aspecto de vulnerabilidade programática ou operacional traduz um contexto preocupante em áreas com elevada endemicidade, como no caso dos municípios deste estudo, uma vez que amplifica ainda mais os impactos da doença e certamente de doenças tropicais negligenciadas 6,7 .

Diferentes trabalhos demonstram a associação entre a ocorrência de hanseníase e comunidades ou territórios negligenciados 21,22,23. No Brasil, uma pesquisa conduzida em um centro de referência no Rio de Janeiro identificou maior prevalência de hanseníase em contatos que se tornaram casos (coprevalentes) em contextos de baixa escolaridade 24 . A maioria da população incluída neste estudo possuía critérios de elegibilidade para a transferência de renda via programa Bolsa Família, apesar de nem todos receberem, trazendo discussões importantes não apenas sobre cobertura, mas também da sua sustentabilidade.

Em um estudo desenvolvido com o objetivo de avaliar o impacto do programa Bolsa Família e do Programa Saúde da Família (PSF) nas taxas de detecção da doença no país, verificou-se que o programa Bolsa Família esteve associado significativamente à redução da taxa de detecção e o PSF esteve associado ao aumento significativo da mesma 21. Esses resultados demonstram um efeito dose/ 
Tabela 1

Caracterização sociodemográfica da população do estudo nos municípios de Rolim de Moura (Rondônia, coorte 2001-2013) e de Picos (Piauí, coorte 2001-2014), Brasil.

\begin{tabular}{|c|c|c|c|}
\hline Variáveis & $\begin{array}{c}\text { Rolim de Moura } \\
n(\%)\end{array}$ & $\begin{array}{l}\text { Picos } \\
\text { n (\%) }\end{array}$ & $\begin{array}{l}\text { Total } \\
\mathrm{n}(\%)\end{array}$ \\
\hline Município & $150(34,3)$ & $287(65,7)$ & $437(100,0)$ \\
\hline \multicolumn{4}{|l|}{ Sexo } \\
\hline Masculino & $55(36,7)$ & $147(51,2)$ & $202(46,2)$ \\
\hline Feminino & $95(63,3)$ & $140(48,8)$ & $235(53,8)$ \\
\hline \multicolumn{4}{|l|}{ Raça/Cor * } \\
\hline Branca & $50(33,8)$ & $65(22,8)$ & $115(26,6)$ \\
\hline Parda & $78(52,7)$ & $152(53,3)$ & $230(53,1)$ \\
\hline Negra & $19(12,8)$ & $61(21,4)$ & $80(18,5)$ \\
\hline Amarela & $1(0,7)$ & $6(2,1)$ & $7(1,6)$ \\
\hline Indígena & $0(0,0)$ & $1(0,4)$ & $1(0,2)$ \\
\hline \multicolumn{4}{|l|}{ Faixa etária (em anos) } \\
\hline$<15$ & $5(3,3)$ & $4(1,4)$ & $9(2,1)$ \\
\hline $15-40$ & $40(26,7)$ & $68(23,7)$ & $108(24,7)$ \\
\hline $41-60$ & $70(46,7)$ & $115(40,1)$ & $195(44,6)$ \\
\hline$>60$ & $35(23,3)$ & $100(34,8)$ & $125(28,6)$ \\
\hline \multicolumn{4}{|l|}{ Situação de moradia * } \\
\hline Moradia definitiva & $136(91,3)$ & $231(80,5)$ & $367(84,1)$ \\
\hline Moradia temporária & $13(8,7)$ & $50(17,4)$ & $63(14,5)$ \\
\hline Assentamento & $0(0,0)$ & $2(0,7)$ & $2(0,5)$ \\
\hline Outra & $0(0,0)$ & $4(1,4)$ & $4(0,9)$ \\
\hline \multicolumn{4}{|l|}{ Escolaridade * } \\
\hline Sem estudo & $25(16,9)$ & $63(22,4)$ & $88(20,5)$ \\
\hline Ensino Fundamental & $80(54,0)$ & $125(44,5)$ & $205(47,8)$ \\
\hline Ensino Médio & $34(23,0)$ & $40(14,2)$ & $74(17,3)$ \\
\hline Ensino Superior & $9(6,1)$ & $53(18,9)$ & $62(14,4)$ \\
\hline \multicolumn{4}{|c|}{ Número de moradores por domicílio } \\
\hline $0-3$ & $105(70,0)$ & $0(0,0)$ & $105(24,0)$ \\
\hline 4 ou mais & $45(30,0)$ & $287(100,0)$ & $332(76,0)$ \\
\hline \multicolumn{4}{|c|}{ Renda total do domicílio (salário mínimo) ** } \\
\hline$<1$ & $68(55,7)$ & $83(33,9)$ & $151(41,1)$ \\
\hline $1-2$ & $28(23)$ & $119(48,6)$ & $147(40,1)$ \\
\hline$>2$ & $26(21,3)$ & $43(17,5)$ & $69(18,8)$ \\
\hline \multicolumn{4}{|c|}{ Mudança da renda após a hanseníase * } \\
\hline Não & $102(69,4)$ & $256(89,2)$ & $358(82,5)$ \\
\hline Sim, com redução & $41(27,9)$ & $25(8,7)$ & $66(15,2)$ \\
\hline Sim, com aumento & $4(2,7)$ & $6(2,1)$ & $10(0,2)$ \\
\hline \multicolumn{4}{|l|}{ Programa Bolsa Família * } \\
\hline Não & $121(82,3)$ & $188(65,5)$ & $309(71,2)$ \\
\hline Sim & $26(17,7)$ & $99(34,5)$ & $125(28,8)$ \\
\hline
\end{tabular}

* Valores ignorados: Raça/Cor - Rolim de Moura = 2 e Picos = 2; Situação de moradia: Rolim de Moura = 14; Mudança de renda após a hanseníase - Rolim de Moura = 3; programa Bolsa Família - Rolim de Moura = 3.

** Valor de referência do salário mínimo: $\mathrm{R} \$ 954,00$. 
Tabela 2

Sobreposição de casos de hanseníase na mesma rede de convívio domiciliar por variáveis sociodemográficas e econômicas nos municípios de Rolim de Moura (Rondônia, coorte 2001-2013) e de Picos (Piauí, coorte 2001-2014), Brasil.

\begin{tabular}{|c|c|c|c|c|c|c|}
\hline \multirow[t]{3}{*}{ Indicadores } & \multicolumn{2}{|c|}{ Sobreposição } & \multirow[t]{3}{*}{ Valor de $p$} & \multirow[t]{3}{*}{$\mathbf{R P}$} & \multirow[t]{3}{*}{ IC95\% } & \multirow[t]{3}{*}{ Valor de $p$} \\
\hline & Sim & Não & & & & \\
\hline & n (\%) & n (\%) & & & & \\
\hline \multicolumn{7}{|l|}{ Município } \\
\hline Rolim de Moura & $98(43,2)$ & $52(24,8)$ & 0,0000 & Referência & - & - \\
\hline Picos & $129(56,8)$ & $158(75,2)$ & & 1,45 & $1,22-1,73$ & 0,0001 \\
\hline \multicolumn{7}{|l|}{ Sexo } \\
\hline Masculino & $92(40,5)$ & $110(52,4)$ & 0,013 & Referência & - & - \\
\hline Feminino & $135(59,5)$ & $100(47,6)$ & & 0,79 & $0,66-0,96$ & 0,0130 \\
\hline \multicolumn{7}{|l|}{ Raça/Cor * } \\
\hline Branca & $61(27,3)$ & $54(25,7)$ & 0,797 & Referência & - & - \\
\hline Parda & $120(53,2)$ & $110(52,4)$ & & 1,02 & $0,82-1,26$ & 0,8788 \\
\hline Negra & $39(17,5)$ & $41(19,5)$ & & 1,09 & $0,82-1,44$ & 0,5552 \\
\hline Amarela & $3(1,4)$ & $4(1,9)$ & & 1,59 & $0,50-4,99$ & 0,3461 \\
\hline Indígena & $0(0,0)$ & $1(0,5)$ & & 0,00 & $0,00-0,00$ & 0,2902 \\
\hline \multicolumn{7}{|l|}{ Faixa etária (em anos) } \\
\hline$<15$ & $4(1,8)$ & $5(2,4)$ & 0,463 & 1,31 & $0,62-2,77$ & 0,4184 \\
\hline $15-40$ & $63(27,7)$ & $45(21,4)$ & & Referência & - & - \\
\hline $41-60$ & $94(41,4)$ & $91(41,3)$ & & 1,14 & $0,92-1,42$ & 0,2129 \\
\hline$>60$ & $66(29,1)$ & $69(32,9)$ & & 1,19 & $0,94-1,50$ & 0,1427 \\
\hline \multicolumn{7}{|l|}{ Situação de moradia * } \\
\hline Moradia definitiva & $198(87,2)$ & $169(80,7)$ & 0,100 & Referência & - & - \\
\hline Moradia temporária & $25(11,0)$ & $38(18,2)$ & & 1,35 & $0,99-1,87$ & 0,0363 \\
\hline Assentamento & $2(0,9)$ & $0(0,0)$ & & 0,54 & $0,49-0,59$ & 0,1924 \\
\hline Outra & $2(0,9)$ & $2(1,1)$ & & 1,07 & $0,40-2,88$ & 0,8747 \\
\hline \multicolumn{7}{|l|}{ Escolaridade * } \\
\hline Sem estudo & $42(18,8)$ & $46(22,3)$ & 0,131 & 1,14 & $0,83-1,57$ & 0,3910 \\
\hline Ensino Fundamental & $100(44,8)$ & $105(51)$ & & 1,18 & $0,87-1,62$ & 0,2854 \\
\hline Ensino Médio & $47(21,1)$ & $27(13,1)$ & & 0,86 & $0,65-1,15$ & 0,3046 \\
\hline Ensino Superior & $34(15,3)$ & $28(13,6)$ & & Referência & - & - \\
\hline \multicolumn{7}{|c|}{ Número de moradores por domicílio } \\
\hline $0-3$ & $65(28,6)$ & $40(19,0)$ & 0,019 & Referência & - & - \\
\hline 4 ou mais & $162(71,4)$ & $170(81,0)$ & & 1,27 & $1,05-1,53$ & 0,0191 \\
\hline \multicolumn{7}{|c|}{ Renda total do domicílio (salário mínimo) ** } \\
\hline$<1$ & $63(14,1)$ & $84(12,9)$ & 0,020 & 1,05 & $0,76-1,45$ & 0,7748 \\
\hline $1-2$ & $88(35,2)$ & $63(40,0)$ & & 0,77 & $0,57-1,03$ & 0,0652 \\
\hline$>2$ & $31(50,7)$ & $38(47,1)$ & & Referência & - & - \\
\hline \multicolumn{7}{|c|}{ Mudança da renda após a hanseníase } \\
\hline Não & $185(81,9)$ & $173(83,1)$ & 0,25 & 0,58 & $0,22-1,50$ & 0,1762 \\
\hline Sim, redução da renda & $38(16,8)$ & $28(13,5)$ & & 0,52 & $0,20-1,37$ & 0,1030 \\
\hline Sim, aumento da renda & $3(1,3)$ & $7(3,4)$ & & Referência & - & - \\
\hline \multicolumn{7}{|l|}{ Programa Bolsa Família } \\
\hline Não & $163(72,1)$ & $146(70,2)$ & 0,657 & 0,96 & $0,78-1,17$ & 0,6571 \\
\hline Sim & $63(27,9)$ & $62(29,8)$ & & Referência & - & - \\
\hline
\end{tabular}

IC95\%: intervalo de 95\% de confiança; RP: razão de prevalência.

* Valores ignorados: Raça/Cor $=4$; Situação de moradia $=1$; Escolaridade $=8$.

** Valor de referência do salário mínimo: $\mathrm{R} \$ 954,00$. 
resposta 23 , evidenciando a importância desses programas para o controle de doenças relacionadas à pobreza, como a hanseníase, em que foram avaliados como primordiais 25 .

Tal cenário aponta para o potencial de se investigar fatores relacionados à vulnerabilidade social à luz do contexto de RCD com sobreposição de casos de hanseníase. O seu reconhecimento poderá oportunizar um melhor entendimento dos fatores que favorecem a persistência da endemia, qualificando o planejamento e a implementação das ações de atenção integral às pessoas acometidas e suas famílias 11. Da mesma forma, para a efetivação das diretrizes norteadoras da vigilância de contatos, em especial em contextos socialmente desfavoráveis 1,11, com problemas operacionais em outras ações de controle, como o tratamento 22 .

Os altos percentuais de casos novos na população economicamente ativa é um outro achado importante a ser analisado, dado o poder incapacitante da hanseníase, além do estigma comumente associado. Ampliam-se as possíveis dificuldades econômicas, em particular diante do iminente afastamento do trabalho. São resultados conhecidos e causam preocupação, visto as características da doença. Em um estudo realizado com o objetivo de analisar a assistência das pessoas afetadas por hanseníase no pós-alta nos municípios de Fortaleza e Sobral, Ceará, identificou-se que a média de idade era de 46 anos, e que 88,4\% tinham alguma ocupação antes do tratamento, percentual que caiu para $75 \%$ durante o tratamento e para $73 \%$ no pós-alta 26 .

Outro aspecto importante é o fato de as pessoas em idade economicamente ativa serem mais afetadas e influenciar o acesso aos serviços de saúde. Ademais, é preciso discutir estratégias capazes de alcançar populações de trabalhadores, como a ampliação de horários de atendimento nas unidades de saúde. Estratégias como essas visam, inclusive, a ampliar ações específicas para a população de homens, historicamente menos frequente nos serviços de saúde 27. É importante também a integração das ações de vigilância, prevenção e controle da hanseníase, em especial nas regiões e municípios de maior endemicidade e nas comunidades que vivem sobre maior exposição às diferentes dimensões de vulnerabilidades 28 .

A expressão de risco para a ocorrência de sobreposição de casos, associada ao Município de Picos, pode ser consequência deste município apresentar maiores coeficientes de detecção (33,2/100.000 habitantes para a população geral e 8,9/100.000 habitantes em menores de 15 anos) em relação a Rolim de Moura (27,8/100.000 habitantes para a população geral e 5,7/100.000 habitantes em menores de 15 anos). Vale ressaltar que esses municípios não se diferem no quesito de nível de endemicidade, sendo igualmente classificados como hiperendêmicos (população geral) e de alta endemicidade (menores de 15 anos) segundo critério do Ministério da Saúde 1,8. Esses municípios estão inseridos em estados com endemicidade muito alta, podendo justificar a considerável ocorrência de sobreposição de casos de hanseníase na população do estudo 11,29.

Em pesquisa realizada em RCD em municípios da Bahia, Rondônia e Piauí, verificou-se a maior frequência de sobreposição de casos de hanseníase nos municípios abordados no Estado de Rondônia (Cacoal e Rolim de Moura) em relação aos municípios do Estado da Bahia (Vitória da Conquista e Tremedal), destacando que os municípios de Rondônia, citados, estão entre os municípios do estado e do país com maior ocorrência de casos novos da doença 11. Outro estudo que abordou a sobreposição de casos em núcleos familiares em Vitória, no Espírito Santo, reconheceu que do total de casos de hanseníase diagnosticados, 44,7\% relataram pelo menos mais um caso de hanseníase em seu contexto familiar 30 .

A proposta de um acompanhamento sistemático e longitudinal de RCD com sobreposição de casos de hanseníase é justificada ainda pela existência de estudos que identificaram as mesmas cepas de $M$. leprae entre os familiares de casos de hanseníase, demonstrando que os contatos se comportam como população fundamental de risco acrescido, podendo se comportar também como fonte de infecção 10.

Destaca-se ainda nas análises feitas neste estudo a associação da sobreposição de casos de hanseníase a domicílios com maior número de moradores por residência. Em diferentes cenários endêmicos no mundo, o maior número de pessoas residindo com um caso de hanseníase sem tratamento gera maior número de pessoas expostas a $M$. leprae 11,12,24. O consequente risco acrescido para $\mathrm{o}$ adoecimento 2,10,29 pode ser intensificado pela presença de determinantes sociais de saúde ainda mais desfavoráveis 5,22,23,29. Essas e outras razões apontam para a necessidade de se considerar a influência de fatores ambientais e condições sociais na persistência da dinâmica de transmissão da hanseníase em RCD 5. 
Em uma pesquisa conduzida no Brasil verificou-se forte associação entre a média de pessoas morando no mesmo domicílio que uma pessoa afetada pela hanseníase e a detecção de casos novos 31. Esses achados fortalecem a importância de os domicílios com maior densidade de moradores e que contam com casos de hanseníase serem priorizados pelo serviço da atenção primária à saúde fortalecendo as ações de vigilância de contatos 20 , que se configura como uma estratégia eficiente de detecção precoce de novos casos 31 .

Destaca-se a importância de se estudar a hanseníase com centralidade na pessoa afetada, a fim de identificar toda a RCD. Essa abordagem poderá resultar em um instrumento de análise para a operacionalização estratégica com indicadores de uso local, quando se considera o conceito ampliado de contatos preconizado pelo Ministério da Saúde 1. Ressalta-se, portanto, a importância de se implementar ações 32 que alcancem toda a RCD com sobreposição de casos, em especial, em áreas endêmicas 1,11,33.

Como limitações deste trabalho, cita-se além do uso de dados secundários que levaram a perdas iniciais, mas também o longo período dos cortes temporais do estudo, favorecendo a ocorrência de viés de memória. Para minimizar o seu efeito, buscou-se complementar as informações coletadas junto à população do estudo em diferentes fontes. A despeito dessas limitações, ressalta-se a relevância da abordagem cuidadosa de dois municípios bem distintos (no que condiz a aspectos culturais, climáticos, sociais e de endemicidade) em regiões críticas do país, com uma proposta inovadora com relação à abordagem da hanseníase em territórios reais.

\section{Conclusão}

A significativa magnitude da sobreposição de casos de hanseníase em uma mesma RCD nas realidades onde se desenvolveu o estudo, marcada por possíveis contextos de vulnerabilidade social, reafirma a necessidade da elaboração de políticas que favoreçam melhorias dos aspectos econômicos, culturais e sociais, minimizando a elevada carga de doenças negligenciadas, a exemplo da hanseníase. Essa perspectiva deve ser considerada e integrada às ações de vigilância e controle da hanseníase com priorização operacional nos territórios pelas equipes de atenção básica.

$\mathrm{O}$ estudo permitiu retratar a importância de se desenvolver ações diferenciadas de vigilância dos contatos sob a perspectiva da abordagem centrada no caso referência, considerando a sua RCD. Essa poderá se tornar uma possível estratégia para ampliar a cobertura das ações de vigilância do contato em contextos sob maior risco de adoecimento, em particular em um momento em que o Brasil ampliou a definição de contato 1,2. Por fim, são necessários outros estudos para o melhor entendimento das RCD na abordagem da família e comunidade afetadas por hanseníase, tornando-as um indicador sentinela para a priorização das ações desenvolvidas pela atenção primária à saúdeS e outros pontos de atenção e cuidado à saúde. 


\section{Colaboradores}

A. S. Reis, E. A. Souza e A. N. Ramos Jr. contribuíram na concepção do projeto, análise e interpretação dos dados, redação do artigo, revisão crítica relevante do conteúdo intelectual e aprovação final da versão a ser publicada. A. F. Ferreira contribuiu na análise e interpretação dos dados, redação do artigo, revisão crítica relevante do conteúdo intelectual e aprovação final da versão a ser publicada. J. R. Cruz, O. D. Araújo, S. F. Macedo, G. V. Silva, G. S. M. García, M. A. G. Carneiro e J. C. Barbosa contribuíram na revisão crítica relevante do conteúdo intelectual e aprovação final da versão a ser publicada.

\section{Informações adicionais}

ORCID: Adriana da Silva dos Reis (0000-00025397-1279); Eliana Amorim de Souza (00000002-9653-3164); Anderson Fuentes Ferreira (0000-0002-1816-9459); Jessíca Reco Cruz (00000002-5682-1292); Olívia Dias de Araújo (00000002-9974-4338); Suyanne Freire de Macedo (0000-0002-1450-8628); Gilberto Valentim da Silva (0000-0003-3994-5442); Gabriela Soledad Márdero García (0000-0002-0884-4721); Maria Angélica Gomes Carneiro (0000-0001-9548-6629); Jaqueline Caracas Barbosa (0000-0001-6401-2244); Alberto Novaes Ramos Jr. (0000-0001-7982-1757).

\section{Agradecimentos}

Ao apoio de parcerias locais como: secretarias municipais de saúde, coordenações do programa de controle da hanseníase, equipes de saúde da atenção básica e universidades. Ao apoio e financiamento via: Netherlands Hanseniasis Relief do Brasil (NHR-Brasil), Programa de Apoio à Pós-graduação (PROAP), do Programa de Pós-graduação em Saúde Pública da Universidade Federal do Ceará, Coordenação de Aperfeiçoamento de Pessoal de Nível Superior (Capes, Bolsa de Mestrado - A. S. Reis), e Fundação Cearense de Apoio ao Desenvolvimento Científico e Tecnológico (FUNCAP, Bolsa de Doutorado - G. S. M. García).

\section{Referências}

1. Ministério da Saúde. Diretrizes para vigilância, atenção e eliminação da hanseníase como problema de saúde pública: manual técnico-operacional; 2016. http://lproweb.procempa.com.

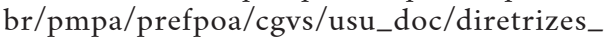
hanseniase.pdf (acessado em 28/Dez/2018)

2. World Health Organization. Global leprosy update, 2017: reducing the disease burden due to leprosy. Wkly Epidemiol Rec 2018; 93:44556.

3. World Health Organization. Estratégia global para hanseníase 2016-2020: aceleração rumo a um mundo sem hanseníase; 2016. http://apps.who.int/iris/bitstream/ handle/10665/208824/9789290225201-pt. pdf? sequence $=17$ (acessado em 28/Dez/2018).

4. Penna ML, Wand-Del-Rey-de-Oliveira ML, Penna G. Spatial distribution of leprosy in the Amazon region of Brazil. Emerg Infect Dis 2009; 15:650-2.

5. Monteiro LD, Mota RMS, Martins-Melo FR, Alencar CH, Heukelbach J. Social determinants of leprosy in a hyperendemic State in North Brazil. Rev Saúde Pública 2017; 51:70.

6. Martins-Melo FR, Carneiro M, Ramos Jr. AN, Heukelbach J, Ribeiro ALP, Werneck GL. The burden of neglected tropical diseases in Brazil, 1990-2016: a subnational analysis from the Global Burden of Disease Study 2016. PLoS Negl Trop Dis 2018; 12:e0006559.

7. Alencar CH, Ramos Jr. AN, Barbosa JC, Kerr LR, de Oliveira MLW, Heukelbach J. Persisting leprosy transmission despite increased control measures in an endemic cluster in Brazil: the unfinished agenda. Lepr Rev 2012; 83:344-53.

8. Ministério da Saúde. Indicadores epidemiológicos e operacionais de hanseníase. Brasil 2001-2017. http://portalms.saude.gov.br/ images/pdf/2018/julho/13/Indicadores-epi demiologicos-e-operacionais-de-hanseniase -Brasil-2001-a-2017.pdf (acessado em 25/Set/ 2018)

9. Romero-Montoya M, Beltran-Alzate JC, Cardona-Castro N. Evaluation and monitoring of the transmission of Mycobacterium leprae in household contacts of leprosy patients in Colombia. PLoS Negl Trop Dis 2017; 11:e0005325.

10. Araujo S, Freitas LO, Goulart LR, Goulart IM. Molecular evidence for the aerial route of infection of Mycobacterium leprae and the role of asymptomatic carriers in the persistence of leprosy. Clin Infect Dis 2016; 63:1412-20.

11. Boigny RN, Souza EA, Romanholo HSB, Araújo OD, Araújo TME, Carneiro MAG, et al. Persistência da hanseníase em redes de convívio domiciliar: sobreposição de casos e vulnerabilidade em regiões endêmicas no Brasil. Cad Saúde Pública 2019; 35:e00105318.

12. Moura ML, Dupnik KM, Sampaio GA, Nóbrega PF, Jeronimo AK, do Nascimento-Filho JM, et al. Active surveillance of Hansen's disease (leprosy): importance for case finding among extra-domiciliary contacts. PLoS Negl Trop Dis 2013; 7:e2093. 
13. Instituto Brasileiro de Geografia e Estatística. Panorama da cidade Picos, Piauí. https:// cidades.ibge.gov.br/brasil/pi/picos/panorama (acessado em 28/Dez/2018)

14. Instituto Brasileiro de Geografia e Estatística. Panorama da cidade Rolim de Moura, Rondônia. https://cidades.ibge.gov.br/brasil/ro/ rolim-de-moura/panorama (acessado em 28/ Dez/2018)

15. Ministério da Saúde. E-Gestor atenção básica: informação e gestão da atenção básica. https:// egestorab.saude.gov.br/paginas/acessoPubli $\mathrm{co} /$ relatorios/relHistoricoCoberturaAB.xhtml (acessado em 28/Dez/2018).

16. Federação das Indústrias do Estado do Rio de Janeiro. Índice FIRJAN de Desenvolvimento Municipal (IFDM); 2013. http://www.firjan. com.br/ifdm/consulta-ao-indice/ifdm-indicefirjan-de-desenvolvimento-municipal-resul tado.htm? UF $=$ PI\&IdCidade $=220800 \&$ Indica dor $=1 \& A n o=2013($ acessado em 28/Dez/2018)

17. Atlas do Desenvolvimento Humano no Brasil. Picos, PI. http://atlasbrasil.org.br/2013/pt/ perfil_m/picos_pi (acessado em 28/Dez/2018)

18. Atlas do Desenvolvimento Humano no Brasil. Rolim de Moura, RO. http://atlasbrasil. org.br/2013/pt/perfil_m/rolim-de-moura_ro (acessado em 28/Dez/2018)

19. Ayres JRCM, França Júnior I, Calazans GJ, Saletti Filho HC. O conceito de vulnerabilidade e as práticas de saúde: novas perspectivas e desafios. In: Czeresnia D, Freitas CM, organizadores. Promoção da Saúde: conceitos, reflexões, tendências. Rio de Janeiro: Editora Fiocruz; 2003; p. 117-39.

20. Romanholo HSB, Souza EA, Ramos Júnior AN, Kaiser ACGCB, Silva IO, Brito AL, et al. Surveillance of intradomiciliary contacts of leprosy cases: perspective of the client in a hyperendemic municipality. Rev Bras Enferm 2018; $1: 163-9$.

21. Nery JS, Pereira SM, Rasella D, Penna ML, Aquino R, Rodrigues LC, et al. Effect of the Brazilian conditional cash transfer and primary health care programs on the new case detection rate of leprosy. PLoS Negl Trop Dis 2014; 8:e3357.

22. Lopes VAS, Rangel EM. Hanseníase e vulnerabilidade social: uma análise do perfil socioeconômico de usuários em tratamento irregular. Saúde Debate 2014; 38:817-29.

23. Lana FCF, Davi RFL, Lanza FM, Amaral EP. Detecção da hanseníase e Índice de Desenvolvimento Humano dos municípios de Minas Gerais, Brasil. Revista Eletrônica de Enfermagem 2009; 11:539-44.
24. Sales AM, Ponce de Leon A, Düppre NC, Hacker MA, Nery JA, Sarno EN, et al. Leprosy among patient contacts: a multilevel study of risk factors. PLoS Negl Trop Dis 2011; 5:e1013.

25. Medley GF, Crump RE, Lockwood DNJ. Interpreting data in policy \& control: the case of leprosy. Indian J Med Res 2017; 145:1-3.

26. Barbosa JC, Ramos Junior AN, Alencar OM, Pinto MSP, Castro CGJ. Atenção pós-alta em hanseníase no Sistema Única de Saúde: aspectos relativos ao acesso na região Nordeste. Cad Saúde Colet (Rio J.) 2014; 22:351-8.

27. Souza EA, Ferreira AF, Boigny RN, Alencar $\mathrm{CH}$, Heukelbach J, Martins-Melo FR, et al. Leprosy and gender in Brazil: trends in an endemic area of the Northeast region, 20012014. Rev Saúde Pública 2018; 52:20.

28. Freitas LRS, Duarte EC, Garcia LP. Analysis of the epidemiological situation of leprosy in an endemic area in Brazil: spatial distribution in the periods 2001-2003 and 2010-2012. Rev Bras Epidemiol 2017; 20:702-13.

29. Bakker MI, Hatta M, Kwenang A, van Mosseveld P, Faber WR, Klatser PR, et al. Risk factors for developing leprosy - a population-based cohort study in Indonesia. Lepr Rev 2006; 77:48-61.

30. Deps PD, Guedes BV, Bucker Filho J, Andreatta MK, Marcari RS, Rodrigues LC. Characteristics of known leprosy contact in a high endemic area in Brazil. Lepr Rer 2006; 77:34-40.

31. Castro SS, Santos JPP, Abreu GB, Oliveira VR, Fernandes LFRM. Leprosy incidence, characterization of cases and correlation with household and cases variables of the Brazilian states in 2010. An Bras Dermatol 2016; 91:28-33.

32. Monteiro LD, Lopes LSO, Santos PRD, Rodrigues ALM, Bastos WM, Barreto JA. Tendências da hanseníase após implementação de um projeto de intervenção em uma capital da Região Norte do Brasil 2002-2016. Cad Saúde Pública 2018; 34:e0007818.

33. Bakker MI, Hatta M, Kwenang A, Faber WR, van Beers SM, Klatser PR, et al. Population survey for determination of risk factors for Mycobacterium leprae transmission and infection. Int J Epidemiol 2004; 33:1329-36. 


\section{Abstract}

The study aimed to analyze the magnitude and sociodemographic profile of leprosy cases linked to household contact networks with overlapping of the disease in two Brazilian municipalities (counties), one in the North and the other in the Northeast, from 2001 to 2014. This was a cross-sectional, descriptive, analytical study based on primary and secondary data from new leprosy cases in Picos, Piaui State, and Rolim de Moura, Rondônia State. "Overlapping" was defined as new cases with the occurrence of at least one more leprosy case in the individual's household contact network. Each household contact network consisted of the index case (first reported case), co-prevalent cases (contacts that became new cases), and household contacts of the index case. A total of 437 new cases treated by the INTEGRAHANS North-Northeast and Piaui projects were assessed, of which 287 (65.7\%) were in Picos and 150 (34.3\%) in Rolim de Moura. Of all the cases assessed, 129 (44.9\%) in Picos and 98 (65.3\%) in Rolim de Moura reported overlapping. There were proportionally more females in Rolim de Moura $(n=95,63.3 \%)$ and males in Picos $(n=147,51.2 \%)$; in the 41-60-year age bracket (Rolim de Moura $n=70,46.7 \%$; Picos $n=115,40.1 \%$ ); with elementary schooling (Rolim de Moura $n$ =80, 54\%; Picos $n=125$, 44.5\%); and individuals living in the same household with up to 3 persons in Rolim de Moura $(n=105,70 \%)$ and with more than 4 persons in Picos $(n=287$, 100\%). Overlapping of leprosy cases in household contact networks was considerably high in the contexts analyzed here, displaying aspects of expanded vulnerability. This perspective should be considered and integrated into leprosy surveillance and control activities.

Leprosy; Epidemiological Monitoring; Infectious Disease Transmission

\section{Resumen}

El objetivo fue analizar la magnitud y el perfil sociodemográfico de los casos de hanseniasis vinculados a Redes de Convivencia Domiciliaria (RCD) con superposición de la enfermedad en dos municipios brasileños, uno de la región norte y otro de la nordeste, de 2001 a 2014. Estudio transversal, descriptivo y analítico, a partir de datos primarios y secundarios de casos nuevos de lepra con residentes en Picos, en el Estado de Piauí, y en Rolim de Moura, en el Estado de Rondônia. "Superposición" fue definida como casos nuevos con ocurrencia de por lo menos más de un caso de lepra en su $R C D$. Cada RCD estaba compuesta por un caso referencia (primer caso notificado), casos coprevalentes (contacto que se convirtió en casos nuevos) y contactos domiciliarios de caso referencia. Un total de 437 casos nuevos abordados por los proyectos INTEGRAHANS Norte/Nordeste y Piauí fueron evaluados, $287(65,7 \%)$ en Picos y $150(34,3 \%)$ en Rolim de Moura. Del total de casos evaluados, 129 $(44,9 \%)$ en Picos y 98 (65,3\%) en Rolim de Moura informaron la ocurrencia de superposición. Se verificó una mayor frecuencia de personas del sexo femenino en Rolim de Moura $(n=95,63,3 \%)$ y masculino en Picos ( $n=147,51,2 \%)$; en la franja etaria entre 41-60 años de edad (Rolim de Moura $n=70,46,7 \%$; Picos $n=115,40,1 \%)$; con enseñanza fundamental (Rolim de Moura $n=80,54 \%$; Picos $n=125,44,5 \%)$ y que vivía en el mismo domicilio con hasta 3 personas en Rolim de Moura $(n=105,70 \%)$ y con más de 4 personas en Picos $(n=287,100 \%)$. La superposición de casos de lepra en $R C D$ presentó una magnitud considerable en los contextos analizados, demostrando aspectos de vulnerabilidad ampliada. Esta perspectiva debe ser considerada e integrada en las acciones de vigilancia y control de la lepra.

Lepra; Monitoreo Epidemiológico; Transmisión de Enfermedad Infecciosa
Recebido em 23/Jan/2019

Versão final reapresentada em 31/Mar/2019

Aprovado em 02/Jul/2019 